\title{
Exploration of older drivers interaction with conversation assistant
}

\author{
Jakub Berka, Lukas Chvatal, Zdenek Mikovec \\ Faculty of Electrical Engineering, \\ Czech Technical University in Prague \\ Prague, Czech Republic \\ berkajak@fel.cvut.cz, chvatlu2@fel.cvut.cz,xmikovec@fel.cvut.cz
}

\begin{abstract}
The number of older drivers will be increasing therefore, their needs and requirements need to be taken into account when designing in-car user interfaces. Current solutions in car industry tend to use big touch screens for controlling the secondary tasks, such as navigation. These solutions are proved to be very distracting while driving. We present a design of conversational assistant for older drivers to improve secondarytask performance, help with decision making in primary tasks with reduced stress. We conducted a user study in the laboratory $(N=7)$ and gained initial knowledge about how the conversational assistant should support older drivers in secondary tasks. Our exploration revealed potential opportunities for the future design of such in-car assistants.
\end{abstract}

Index Terms-Driving, Voice Interfaces, Multitasking, User Centered Design

\section{INTRODUCTION}

$\mathbf{T}$ HE number of older drivers will be increasing, as the percentage population aged 65 and over was $13,8 \%$ in 2000 and $18,8 \%$ in 2017 , it is expected that it will rise to $24 \%$ by the year 2030 [1]. Whatmore, seniors use individual car transport much more compared to the past [2].

With growing age drivers start to experience more frequent problems with their visual perception, attention and fast decision making [3].

Unrelated to the driver's age, all drivers are exposed to a steadily increasing number of distractors. According to [4] and [5] distractors can cause secondary task distraction, which diverts the driver's attention away from the primary task driving. The driver becomes occupied with events which are unrelated to driving, occur away from the forward roadway and urge the driver also to look away from the forward roadway. We can divide these distractors into two groups - internal distractors (inside the vehicle) and external distractors (objects located outside the vehicle). In this paper, we are investigating only the internal distractors, which in our case are navigation system and messaging while driving. The distraction has a significant contribution to driving accidents [4] (23\% of all crashes and near-crashes are caused by secondary task distraction). According to [6] the potential for a secondary task to distract the driver is determined by the task complexity, current driving demands, driver experience and skills, as well as driver's willingness to engage in the task

The influence of distracting tasks on driving performance is bigger for more complex activities, especially when drivers are older [7]. Therefore our goal is to reduce the secondary task complexity and workload with the help of a conversational assistant. It is now possible to implement conversational assistants into cars, thanks to the progress in speech recognition systems in recent years. Also with the arrival of car-tocar and car-to-infrastructure communication, these intelligent assistants can become safer and less distracting, as they will take into account broader context, for instance, road situation, nearby traffic, etc.

In this paper, we present results from a Wizard of Oz user study with older drivers where we explored the experiences with conversational assistant designed for older drives to help them handle the secondary tasks (navigation task and messaging task) with maintaining the safety and lowest distraction as possible. Our goal was to explore how such system as a conversational assistant will be accepted by older drivers and what benefits it could bring them. Our design evoked various reactions: some drivers found the conversation with the system while driving still too distracting, but some were satisfied with our design mainly in messaging task where our proposed semiautonomous messaging system was rated very positively.

\section{RELATED WORK}

In this section, we examine existing solutions and concepts related to secondary tasks, distractions and use of conversation interaction while driving. We focused mainly on studies where the target groups were mostly older people or where the studies aimed at problems related to our case of study.

\section{A. Distractions while driving}

Distraction can be often caused by other persons - passengers or someone on the phone, but the effect is not usually the same in both cases. Passenger is a direct participant in traffic so the conversation can be modified according to the situation on the road. In opposite, a phone call cannot be naturally suppressed according to conversation suppression hypothesis [8]. Bruyas et al. rescheduled these real-time tasks to become asynchronous. Their results show that it can help to reduce pressure on driver compared to synchronous phone communication, as a suitable place can be chosen in respect of traffic situation. Another most common problem is manual (frequently finger touches on the screen) control of infotainment systems which leads to long or frequent off-road glances 
causing great danger. The study by Lee et al. [9] examines how errors in interacting with infotainment systems influence driving performance, specifically input of the words using a touchscreen, and how drivers recover from these errors. They suggest that for preventing high distraction caused by secondary task, sufficient but not greater than necessary visual information should be provided.

Although the speech-based interface has also some negative impact on driver's workload, according to Maciej and Vollrath [10] it is still better than displays and manual controls.

\section{B. Multimodal Interaction in the Car}

One possible approach to overcome the limitations of speech-only interfaces can be multimodal interaction, which may provide fine-grained control with immediate feedback and easy undo of actions. Pfleging et al. [11] designed an interaction that combines speech and gestures on the steering wheel. To adjust distraction when interacting with infotainment systems some novel interactions techniques are becoming popular, e.g. mid-air gestures. However, the problem is with the feedback, which is still mainly through visual displays, Shakeri et al. [12] investigated different types of feedback modalities. Their study concludes that non-visual feedback (auditory, tactile) can significantly reduce distraction. In the work of Tashev et al. [13] authors created multimodal dialog system for infotainment and also formulated key requirements for voice enable infotainment systems, concerning the efficiency of multimodal interaction during high cognitive load situations.

\section{Older drivers}

It is obvious that older drivers have special demands. Besides some physical impairments (visual, motion, etc.), they are more sensitive to time pressure and complexity of the tasks. On the other hand, older drivers are calmer, less reckless and less daring drivers than earlier in life and there are many aspects from which older drivers can profit. For example from their life-long driving experience, maturity and flexibility to drive at times and places that they perceive as being safer [6]. According to Bjelkemyr et al. [14] this flexibility closely relates to a phenomenon of self-regulation, which can manifest as avoiding certain conditions (e.g. driving at night or during rush hour) or difficult traffic situations (e.g. driving through specific intersections), next reducing speed, avoiding motorways, big cities, long distance travels and avoiding unknown cities, etc.

\section{Secondary tasks}

There is a wide variety of secondary tasks that can be performed while driving. For our research, we have chosen two tasks: navigation on the unknown route and messaging as they are highly distracting the driver [15]. The navigation system is common equipment in cars with a lot of useful functions nowadays, but design low-distracting user interface is not an easy task, many drivers also use their mobile phones as a navigation aid. Use of mobile phones (hand-held) in many European countries is prohibited, but drivers keep using it. Hands-free use is also proven to be distracting while driving [16]. On the other hand, there are cases in which phone calling or sending messages is necessary for drivers, therefore they need to be supported by intelligent systems to finish these tasks safely.

1) Navigation: The navigation task is typically supported by navigation systems that heavily rely on the visual (displays with maps) and physical (touch screen with user controls) interaction. The distraction level, especially in some stressful situation is very high [6], [10], [14]. For the purposes of an experiment, we focused on dealing with stressful error situations. According to research by Bjelkemyr et al. [14] older people reported to be calmer, less reckless and less daring drivers than earlier in life, but less busy roads are more important to them than the duration of the trip, because of their time-flexibility [6]. Bjelkemyr et al. also found out they have problems in finding their final destination and need to plan their travel in advance. Whatmore, mental states during driving can influence mood after arrival (stress), the passenger is often used as a co-pilot when in some stressful traffic situation. The researchers conclude that support systems for older drivers should increase comfort and decrease their level of stress.

2) Messaging: For the messaging task, there are several insights and notes that we took into account. The use of a mobile phone and even the hands-free phone calls while driving are proven by research to be very distracting and dangerous secondary tasks [16]. Atchley et al. [17] examined interview with 348 young responders, and the results show that sending, replying and reading of text messages have been recognized as riskier behavior compared to talking on the mobile phone. On the other hand, when having a mobile phone conversation driver's reaction time is increasing with the increased time of the conversation [18]. Moreover, according to Dula et al. [19] more emotional and intense phone conversations tends to cause more dangerous driving behaviors. Fofanova and Vollrath [6] also state that older people have worse driving performance when using a mobile phone. According to Lipovac et al. [16] with the growing age, the percentage of those who considered mobile phone use while driving an unsafe activity increased.

\section{DESIGN}

In this section, we propose a concept of conversational assistant designed for older drivers. For the beginning of our research, we focused only at two selected tasks and the design process started with the following scenarios.

\section{A. Dealing with error stressful situation.}

This scenario covers the recovery from error and stressful situations (see sections III-A1 and III-A2). The error situations can be identified either by the system, or they can be identified by the user itself. When the system identifies this situation, the driver will be informed about it. Then the driver can confirm or refuse that the situation is erroneous. The system then will suggest the driver how to solve the situation. Furthermore, the driver can modify the suggested solution or accept it. 
TABLE I

EXAMPLE 1 OF THE DiALOG BETWEEN THE USER (U) AND THE CONVERSATIONAL ASSISTANT (A) FOR SCENARIO 1.

A: It seems that is hard to turn left now, is this true?

U: Yes, I can't make it.

A: That is no problem, you can continue straight this lane, there is another way and the delay will be only one minute.

U: Okay, that's good.

A: After the crossroad, try to get to the left lane when it is possible. You will be informed about next steps, don't worry.

TABLE II

EXAMPLE 2 OF THE DIALOG BETWEEN THE USER (U) AND THE CONVERSATIONAL ASSISTANT (A) FOR SCENARIO 1.

A: It seems that it is hard to turn left now, is this true?

U: Yes, I can't make it.

A: That is no problem, you can continue straight this lane, there is another way and the delay will be only one minute.

U: I rather turn right, the situation in front of me looks complicated.

A: Okay, turn right. There is a better way, which is more peaceful and the delay is insignificant - only 4 minutes more. You have plenty of time to get to your final destination.

1) Wrong lane on a crossroad: The driver is arriving at the crossroad with three lanes. Traffic is heavy, s/he is in the most right lane but according to the navigation, s/he should be in the most left lane to turn left. System evaluated that $\mathrm{s} / \mathrm{he}$ is too close to the crossroad to change the lane safely (see dialog examples in I, II and III).

TABLE III

EXAMPLE 3 OF THE DIALOG BETWEEN THE USER (U) AND THE CONVERSATIONAL ASSISTANT (A) FOR SCENARIO 1.

A: It seems that is hard to turn left now, is this true?

U: No, I just want to turn right to the fast food on the corner.

A: Okay, do you mean "McDonald's" restaurant?

$\mathrm{U}$ : Yes.

A: Perfect, go ahead and the parking lot is in 200 meters. The entrance to the drive thru is few meters further.

2) Lack of fuel: Driver noticed that a gasoline indicator shows that there is no much gasoline in the tank, feels insecure, thus starts conversation with the system (see dialog example in IV).

\section{B. Semi-autonomous messaging system}

For our first design iteration of messaging task we selected model situation described in the following scenario. The scenario represents situation when the driver wants to send informative sms messages about the journey to someone (e.g. a partner, relatives). See dialog example in V.

It is Thursday morning and in a town's swimming pool ends regular lecture for seniors. One of the participants is
TABLE IV

EXAMPLE 4 - EXCERPT FROM THE DIALOG BETWEEN THE USER (U) AND THE CONVERSATIONAL ASSISTANT (A) FOR SCENARIO 1.

U: I have a low amount of fuel, is it enough to get home?

A: It should be enough to get home, but I recommend to stop at petrol station due to the traffic situation. Do you want to find petrol stations?

$\mathrm{U}$ : Yes, find the stations.

A: There is a petrol station OMW twelve kilometers ahead on the road you are going. Do you want to stop there?

U: Nah, is there any shell station? I have their VIP card.

TABLE V

EXCERPT FROM THE DIALOG BETWEEN THE USER (U) AND THE CONVERSATIONAL ASSISTANT (A) FOR SCENARIO 2.

U: I want to write an sms.

A: I see that you are driving home, do you want to set sending sms to Peter (husband) or someone else?

U: To Peter, please.

A: Got it. Should I send him that you are about to go home?

$\mathrm{U}$ : Yes.

A: Do you want to add something to the message?

$\mathrm{U}: \mathrm{No}$.

A: Okay. I have sent the message to Peter. Maria had started driving but after few kilometers she got stuck in a traffic jam.

A: It seems that there will be a little delay due to this traffic jam.

Should I send a message to Peter about it?

U: Yes, please.

A: I will send him this text:

"Hi Peter, I am in a traffic jam on the Neibolt street, it seems that I will arrive ten minutes later, around 6:45. Maria."

Should I add something?'

70-year-old Maria, who is an enthusiastic swimmer and she never misses a lecture. She lives with her husband Peter in the nearby city, but the connection by public transport is not good, so she uses car for transportation. Her husband is worried about her drive back as she could be tired after an hour of swimming. Unfortunately, there is no other option. Thankfully, they have got a semi-autonomous messaging system in their car so he can be calmer when gets messages about the journey of his wife. After the engine had started, Maria got information that the messaging system is ready and asked if she wants to send a message to her husband that she is about to go. The system reads a prepared message and asks for confirmation. During the way home, Maria got into the rush hour of a city so got stuck in a traffic jam. In that moment the system asked if she wants to inform her husband about the traffic situation and delay. After her reply, the system sent a message to Maria's husband about the situation and estimated delay and then informed her that the message had been sent. Both Maria and her husband can be relaxed, because they know that everything is alright. 


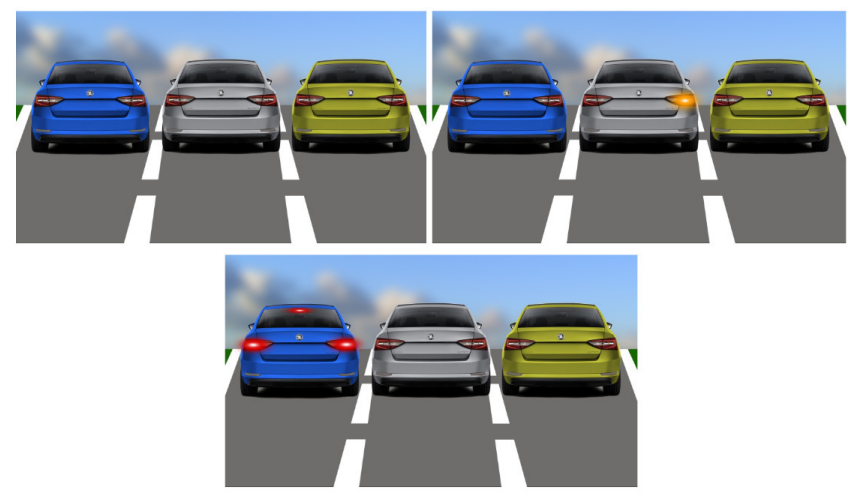

Fig. 1. Screens of primary task script in three different states (none action, middle car signals right turn, left car is braking)

\section{FIRST EXPERIMENT}

\section{A. Participants}

Target group for our experiment were people over 60 years old. Having a currently valid driving licence was not required but the participants should have driving experience. We recruited 7 participants. They were aged from 61 to 74 years $($ mean $=68.71, S D=5.47)$. All of the participants were native Anonymized speakers. For more information see Table VI

\section{B. Apparatus}

Primary task simulation. The primary task was designed for simulation of paying attention while driving. It was being handled by a script, which showed static pictures of three cars (see in Figure 1) from the back view on the computer screen. After a period between 6 to 10 seconds (randomly selected), one of the cars performs action of braking, signals turning right or left (illustrated by turning on back brake lights or blinking of signal lights). All of these parameters are chosen randomly. Participant should react immediately to these actions by pressing the correct key on the keyboard (spacebar - brake lights, left/right arrow for turning signals).

Secondary task. Wizard of $\mathrm{Oz}$ technique was used for simulation of interaction with designed dialogue system for each task. For this experiment moderator played pre-recorded phrases according to the participant's responses and the current state of dialogue. For the uncovered states (unexpected participant's answer or request), universal recovery phrases were prepared. Furthermore, the participant could ask for repetition of question/answer. Playing of pre-recorded phrases was handled by the moderator using the web application (HTML and JS), which allows controlling playback of phrases for specific states. The control interface also contains a dialog state diagram (see example in Fig.2) for the given scenario for better orientation of moderator in the dialog flow.

Equipment. The experiment was done by using two notebooks, the primary task simulation script was running on the first one (with an external keyboard connected) and the second one for controlling the Wizard dialog application by

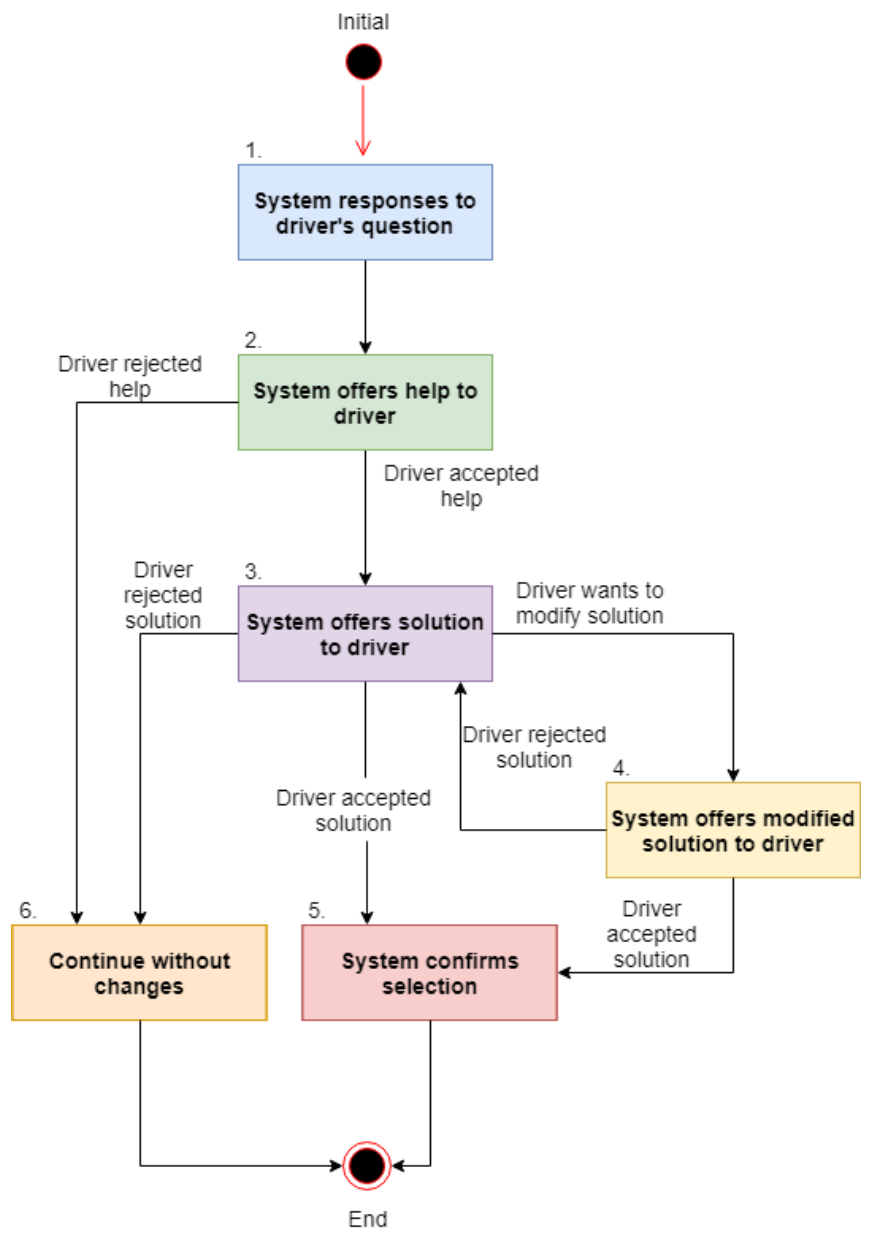

Fig. 2. Dialog state diagram example (Task B1).

the moderator. Each use-case was complemented by a printed map for better illustration of situational context described in the scenario presented to the user. Furthermore, we used a small car model for showing participant's position on the map. Experiment setup (without the moderator's notebook) can be seen in Figure 3.

Data Collection. During each session, the audio was recorded. The reaction time and correctness of the pressed key were also measured. However, the number of participants is not sufficient for quantitative testing and measured values are not significant for this time, it can be used for later experiments or evaluation.

\section{Procedure}

The experiment was divided into the following individual tasks, which were based on previously described navigation and messaging tasks with selected scenarios.

1) Experimental tasks:

- Training: primary task only

- A1: Stressful situation on the crossroads - false negative

- A2: Stressful situation on the crossroads - false positive

- B1: Low fuel 
TABLE VI

TABLE WITH DETAILED INFORMATION ABOUT PARTICIPANTS

\begin{tabular}{|c|c|c|c|c|c|c|c|}
\hline Participant ID & Balancing & Age & Gender & Active driver & Infotainment exp. & Voice interface exp. & Visual Impairment \\
\hline P01 & $\mathrm{AB}$ & 61 & male & yes & yes & yes & $\begin{array}{l}\text { reading and distance } \\
\text { eyeglasses (5-6 dioptre) }\end{array}$ \\
\hline P02 & BA & 62 & female & yes & yes (not using navigation) & no & distance eyeglasses (2 dioptre) \\
\hline P03 & $\mathrm{AB}$ & 74 & male & no & no & no & $\begin{array}{c}\text { uses eyeglasses only when } \\
\text { the light conditions are not good }\end{array}$ \\
\hline P04 & $\mathrm{BA}$ & 74 & male & yes & no & no & reading eyeglasses \\
\hline P05 & $\mathrm{AB}$ & 67 & male & yes & yes & no & no \\
\hline P06 & BA & 72 & male & yes & yes & no & $\begin{array}{l}\text { distance eyeglasses } \\
\text { (2 dioptre) }\end{array}$ \\
\hline P07 & $\mathrm{AB}$ & 71 & female & yes & no & no & $\begin{array}{l}\text { uses eyeglasses for } \\
\text { reading and driving }\end{array}$ \\
\hline
\end{tabular}

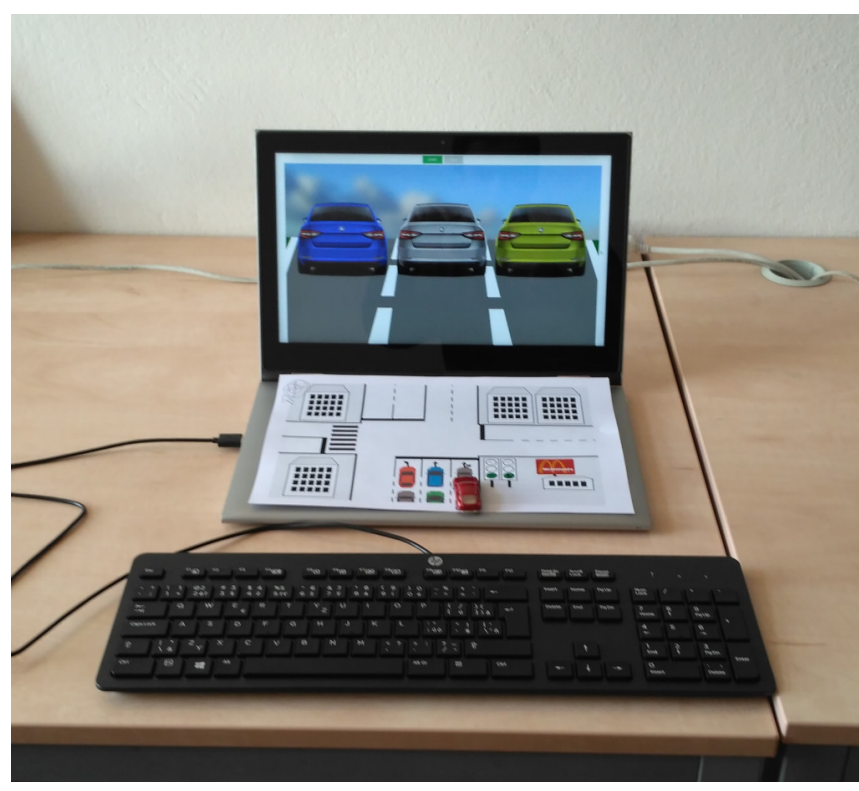

Fig. 3. Experiment setup, without second notebook for experienter

- B2: Semi-autonomous messaging assistant

2) Balancing: For the experiment we chose the balancing of the experimental tasks as $\mathrm{AB}-\mathrm{BA}$. With that every participant will go through Training phase, interacting only with primary task. Task assignment to participants can be seen in the Table VI.

3) Surrounding scenarios: Before the beginning of each task, it was necessary to empathize the participant into the situation. For that we used following surrounding scenarios together with printed maps (map for task A1 and A2 can be seen in the Figure 4, map for tasks B1 and B2 in the Figure 5). After the simulation is started, the moderator initiates interaction with the participant through a dialog system, following the state diagram of the dialog (this is repeated for all tasks).

A1. The driver arrives at a crossroad in the right turn lane. The moderator shows to the participant where his/her final destination is, using the map, and where it is best to get to it (turn left). At the same time, moderator points out that the participant is in the right lane and on the traffic situation in other lanes. The participant begins with an imaginary approach to the intersection and the moderator launches the primary task simulation.

A2. The driver arrives at a crossroad in the right turn lane. The moderator shows to the participant where his/her destination is, using the map, and where it is best to get to it (turn left). At the same time, moderator points out that the participant is in the right lane and on the traffic situation in other lanes. However, the participant is instructed to "make a small break" and stop at a fast food restaurant that is a few meters after turning right. The participant begins with an imaginary approach to the intersection and the moderator launches the primary task simulation.

B1. The driver is on his/her way home. Finding out that he/she does not have too much fuel and many miles ahead of him/her, theoretically, there might not be enough fuel in the tank to complete the trip. The participant is also informed that s/he owns a Shell VIP card, thanks to that a liter of gasoline is much cheaper than the normal price. The participant is instructed to try to deal with a possible fuel shortage. To add more weight to a given situation, s/he is once again informed of the long journey waiting for him/her and the fuel tank indicators, which for the time being are not signaled by the indicator light, but it is clear that this may happen soon. The participant starts when s/he is already on the road and the moderator launches the primary task simulation.

B2. The driver sets out on his/her way home to his son David. S/he is very caring and would like to have an overview of the course of the driver's journey (when he set off, delay, etc.). The moderator informs the participant that it is possible to activate the messaging assistant at the beginning of the trip, which can help him/her to inform the son. It is up to the participants to activate the assistant. Moderator informs the participant that use-case ends after imaginary arrival home. The participant begins the situation when he sits in the vehicle and sets out on the road. Moderator launches primary task simulation. After the simulation is started, the moderator waits for the participant's stimulus and then initiates interaction with the dialog system by following the status diagram of the dialog. If the suggestion does not come for a longer period of time, the moderator initiates the dialogue initiation phrase (in the post-questionnaire asks why the participant did not start the communication). 


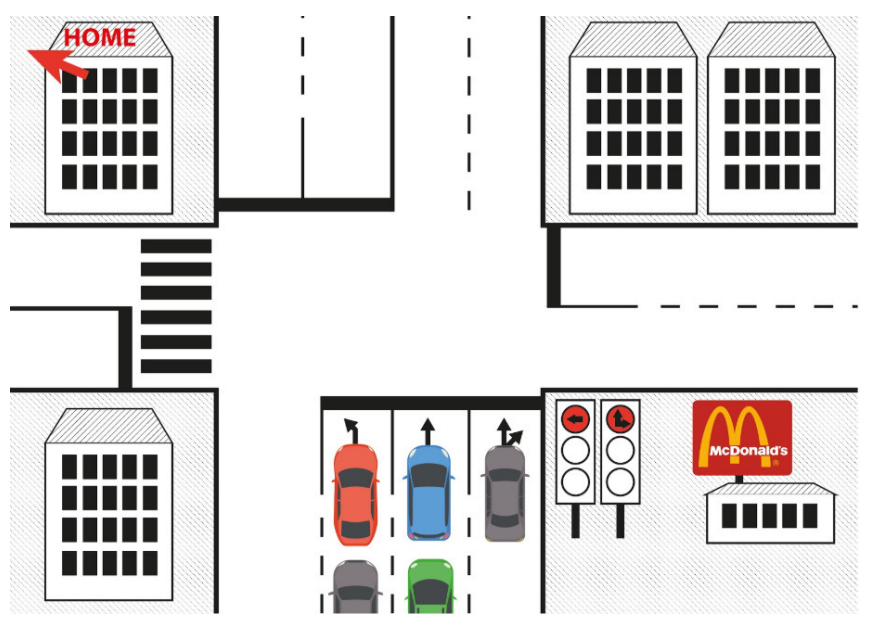

Fig. 4. Map for better illustration of the Navigation scenario

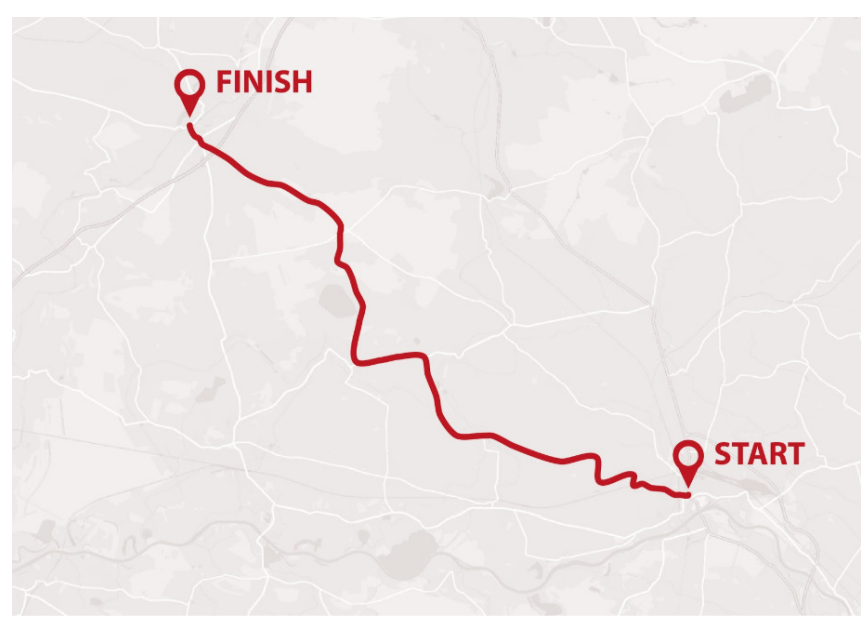

Fig. 5. Map for better illustration of the Navigation scenario and Messaging scenario

4) Post-interviews: Three post-interviews were executed to get feedback from participants about the subject of testing. One cumulative for tasks A1 and A2, and two separate for each B1 and B2 task. The open questions about their first impressions and how did they liked the dialogue with the conversational assistant, were posed to participants. We also asked participants about their subjective judgements about the level of comfort ("I felt comfortable when I was using the system."), comprehension ("I think that the system spoke comprehensively."), intuitive conversation ("I think that the conversation was intuitive.") and level of acceptance in traffic ("I can imagine that I would use the system in traffic.") on a 5 point Likert scale as a level of agreeing with presented statements.

\section{RESUlts}

\section{A. Wrong lane on the crossroad}

Trust and distraction. In general, situation in scenario A was hard to imagine for participants, P07 said she had never been in this situation in the real life, P02 did not trust the advice from the system to continue straight, because it is not sure what will be the situation on the next junction, P03 cannot imagine using the system in this situation. He does not like larger dialogues while driving (also with passengers), he rather focus on driving. P06 said it was excellent, that system reacted immediately to the situation. Regarding the deviation to the restaurant, it understood me and provided me even with the distance information.

Complexity of answers. Participants P01, P02 preferred shorter speech. They answered mostly with short answers, he used answers like: "Yes/Yep/No", etc. , therefore, did not get enough information to go into the right state. On the other hand, P06 answered the first question with a complex utterance ("Yes, but I will change the route to the McDonald's restaurant").

Modification of system suggestion. Some of the participant modified the suggestion from the system, P01 for instance, did not declined or accepted the suggestion from the system, but said: "To the right". Even if he was told he want to turn left to reach the destination, he chooses the third option. P05 replied yes to the question if he cannot turn left ("It seems that is hard go left now, is this true?"). But the answer to the question if he wants to continue straight was: "Not really, we will try it for now."

P04 would like to have more additional information for making his decisions. For instance availability of parking lots, which can be also found on road signs, but they are not so important and he can miss them because he is focusing on the driving.

\section{B. Low fuel situation}

Start of the conversation. P01, P03, P04, P07 did not initiate the dialogue by themselves or were not sure how to initiate it. P04 expected that the system will offer him his prefered petrol station automatically. P05 was continuously talking about the situation (thinking aloud), about worries if there is enough fuel to reach the destination, it would be hard for the system to recognize his intent.

Modification of system suggestion. P01, P05 correctly rejected OMW station as they were instructed they had VIP card for different petrol station. P03 did not ask about searching for his preferred petrol station, he waited for information given by the system. P06 was asking the system to find Shell gas station ("Find closest Shell"). Participant refused offered Shell (because of the deviation from the route) and asked for another Shell gas station. System offers OMW station which is directly on the route. Participant refuses and asked for Shell station ( "How far will be Shell"). System offered the same Shell (with deviation). Participant refused and asked for another Shell. System refused and participant asked why it is not possible to find another Shell Station. Participant tries to refine the request by specifying to find Shell station without deviation ("Find closest Shell without deviation"). Finally participant accepts the Shell station with deviation. P06 disliked the offer of the gas stations. There for sure must be another Shell station 
closer to the route, what is a problem of the database of POIs rather than problem of the dialogue.

General. P01 was often answering before the utterance from system was completed, he seemed to be impatient. P02 used "Thank you" to end the conversation, she said it was great and she liked it, also understood it well. P03 did not like risky situation, when he was not sure if the amount of petrol is enough to get to final destination. P04 did not know that he can ask about his favorite petrol station, so the system was still repeating information about the same petrol station. It was annoying for him. On the other hand, P07 liked the fact that she could ask the system about different petrol stations.

\section{Messaging assistant}

Distraction. P01 mentioned that the sending of messages should be automatic, when he starts the route navigation. P01 also said the amount of the conversation during this scenario was too big. He would like it to be more automatic, because this semi-automatic system can be still too distracting. P03 appreciated the ability to send message without distraction.

Message preparation. Participant P05 dictated all parameters in one sentence (time, contact phone number, message). P06 was dictating the content of the message only and as an appendix adds "...and then I will finish the sentence".

Sending message. On the way P06 asked to send a message to David by means of clear request to the system ("Send $a$ message to David."). Participant requested to add time to arrive to the message.

Complexity of answers. P05: The answer to the question "Do you want to send an SMS" was complex "Well, definitely, because I will be late, I guess.". The answer to the question "Do you want to add something to the SMS" was also complex "No, that is enough, it is exactly what I meant."

General. P02 started conversation or activated the messaging system with "I'm just leaving", system then asked about the purpose of the drive, participant answered "I am going to see you". Participant P03 appreciated using of messaging assistant. P06 requested confirmation of the message delivery.

\section{Post-interview}

P01 said that the system was too verbose. P02 mentioned it would be difficult for her to learn how to use this system, because she does not use the navigation system in her car and she drives only on known routes. On the other hand, she would welcome the messaging system in her car. P03 had doubts about the reliability of these systems in nowadays cars in general. P05 mentioned he was a bit nervous about what and when will happen, it means it is not clear when and what the system will start talking about. "The system could maybe continuously talk about the situation. Long silences makes me nervous if the system is functioning and the question is surprising me." P07 liked that he can control message sending with voice and did not have to use hands or make a phone call.

\section{E. Subjective judgements}

Fig. 6 shows that system acceptance in traffic was positive mainly for tasks B1 and B2, regarding tasks A1, A2 almost half of the participants would not accept this system in real traffic situations. Almost $100 \%$ of participants strongly agreed that the conversation with system was intuitive, only one participant was neutral in task B2. 90\% of participants agreed or strongly agreed that the system spoke comprehensively. 2 participants disagreed in task B2 about the comprehension. $95 \%$ of participants agreed or strongly agreed the system was comfortable to use.

\section{DISCUSSION}

The qualitative results show that drivers often did not know how to start a conversation with the system. First, older drivers have mostly no voice control experience. Second, the tasks were hard to imagine for the participants. It was our intention not to tell them how they should start the conversation before we started the study, and we just wanted to observe their behavior without previous experience with our system.

The complexity of the participant's utterances to the system varied widely across the participants, some were talking to the system in complex sentences so that current natural language understanding systems would have a problem identifying the user's intent. Conversely, there were users who talked to the system very briefly and austere.

The messaging assistant was perceived with a predominantly positive attitude among the participants. They would find it practical and could imagine using it in the car. Some participants would expect the messaging assistant to be even more autonomous and found it unnecessary verbose. Here arises an opportunity to personalize the assistant, for example, based on verbosity and level of automation.

In the navigation task, the biggest problem for the participants was to imagine the scenario situations, but many of them were positive about the contextual information the assistant offered them. The results show that the assistant should be able to offer alternatives, for example, when choosing a gas station. For instance, an assistant informed in advance about the low fuel level, but with the assurance that there is still enough to reach the destination, the participants welcomed and then freely decided whether to stop at the gas station or not. Similarly, in the simulated crossroad situations, drivers freely decided whether to accept the system's recommendations or not.

\section{A. Limitations}

The main limitation of a user study we conducted was the fact that we did not use a high-fidelity driving simulator instead, we used our low-fidelity simulation of the primary task described in the section IV-B. Therefore, some of the participants had a problem to imagine the context in scenarios we presented them. Furthermore, because of our experimental setup, we were not able to simulate real stressful road situations and so we do not claim that our design will reduce the stress of older drivers, but rather see an opportunity 


\section{Subjective judgements}

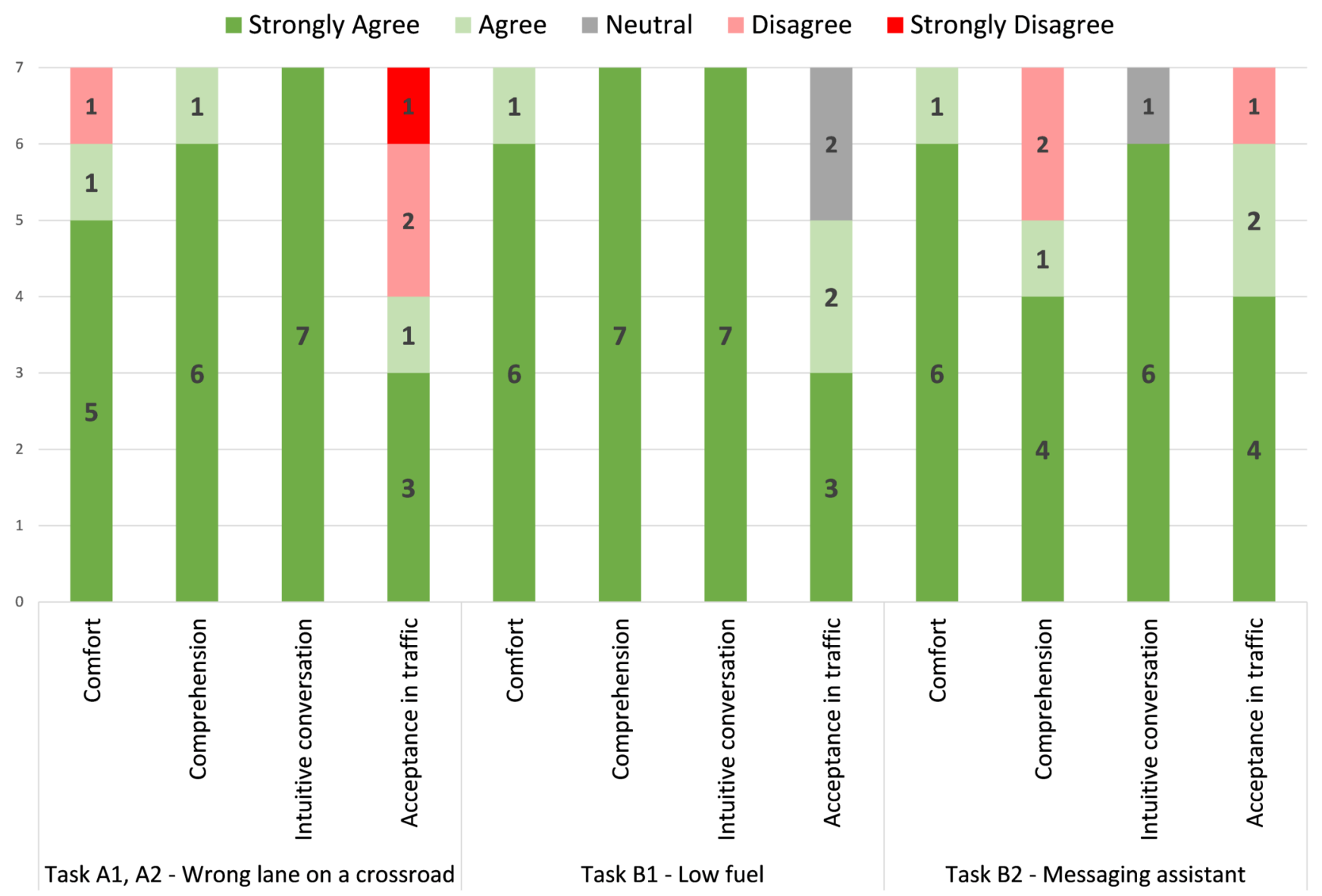

Fig. 6. Subjective judgements about level of comfort, comprehension, intuitive conversation and acceptance in traffic $(N=7)$.

in development such intelligent system which would respect older drivers according to our findings.

\section{B. Future Work}

Given our findings and limitations, future work should furthermore investigate the use of other modalities for supporting the older drivers in tasks that require more rapid action from the driver. For instance in situations on the crossroad and also other fast and stressful situations, the haptic interface, we believe, could be more suitable.

We would also like to further explore the ways how to announce the driver that system is going to talk to him/her, as some participants were surprised when the system started to speak after a longer pause. Again multiple modalities should be investigated. The use of the context of the car, road situation, surrounding traffic or drivers preferences and skills should be explored to determine when is the right time for the system to start a conversation with the driver.

\section{CONCLUSION}

We designed a low-fidelity prototype of conversational assistant for two secondary tasks, navigation and messaging. We also conducted a qualitative user study with 7 older drivers, using the Wizard of $\mathrm{Oz}$ method. The results of our study show that support in secondary tasks for older drivers while driving can be carried out by the conversational assistant. But still, there are some limitations when using only speechbased interface, therefore, the use of other modalities should be investigated.

\section{ACKNOWLEDGMENT}

This research has been supported by grant no. SGS19/178/OHK3/3T/13 (FIS 13139/161/1611937C000) and by project RCI (reg. no. CZ.02.1.01/0.0/0.0/16 019/0000765) supported by EU.

\section{REFERENCES}

[1] Czech Statistical Office, "Seniori," [Online; accessed 13-May-2019]. [Online]. Available: https://www.czso.cz/csu/czso/seniori 
[2] BESIP, "Senior v silnicnim provozu," [Online; accessed 13May-2019]. [Online]. Available: https://www.ibesip.cz/Tematickestranky/Seniori/Senior-v-silnicnim-provozu

[3] H. C. Lee, A. H. Lee, D. Cameron, and C. Li-Tsang, "Using a driving simulator to identify older drivers at inflated risk of motor vehicle crashes," Journal of Safety Research, vol. 34, no. 4, pp. 453 - 459, 2003, senior Transportation Safety and Mobility. [Online]. Available: http://www.sciencedirect.com/science/article/pii/S0022437503000471

[4] S. G. Klauer, T. A. Dingus, V. L. Neale, J. D. Sudweeks, D. J. Ramsey et al., "The impact of driver inattention on near-crash/crash risk: An analysis using the 100-car naturalistic driving study data," 2006.

[5] E. Chan, A. Pradhan, M. Knodler, A. Pollatsek, and D. Fisher, "Evaluation on a driving simulator of the effect of drivers' eye behaviors from distractions inside and outside the vehicle," Human Factors, 2008.

[6] J. Fofanova and M. Vollrath, "Distraction while driving: The case of older drivers," Transportation Research Part F: Traffic Psychology and Behaviour, vol. 14, no. 6, pp. 638 - 648, 2011, special Issue: Driving Simulation in Traffic Psychology. [Online]. Available: http://www.sciencedirect.com/science/article/pii/S1369847811000775

[7] D. Shinar, N. Tractinsky, and R. Compton, "Effects of practice, age, and task demands, on interference from a phone task while driving," Accident Analysis \& Prevention, vol. 37, no. 2, pp. 315 - 326, 2005. [Online]. Available: http://www.sciencedirect.com/science/article/pii/S000145750400096X

[8] M.-P. Bruyas, C. Brusque, S. Debailleux, M. Duraz, and I. Aillerie, "Does making a conversation asynchronous reduce the negative impact of phone call on driving?" Transportation Research Part F: Traffic Psychology and Behaviour, vol. 12, no. 1, pp. 12 - 20, 2009. [Online]. Available: http://www.sciencedirect.com/science/article/pii/S1369847808000594

[9] J. Y. Lee, M. C. Gibson, and J. D. Lee, "Error recovery in multitasking while driving," in Proceedings of the 2016 CHI Conference on Human Factors in Computing Systems, ser. CHI '16. New York, NY, USA: ACM, 2016, pp. 5104-5113. [Online]. Available: http://doi.acm.org/10.1145/2858036.2858238

[10] J. Maciej and M. Vollrath, "Comparison of manual vs. speech-based interaction with in-vehicle information systems," Accident Analysis \& Prevention, vol. 41, no. 5, pp. 924 - 930, 2009. [Online]. Available: http://www.sciencedirect.com/science/article/pii/S0001457509001080

[11] B. Pfleging, S. Schneegass, and A. Schmidt, "Multimodal interaction in the car: Combining speech and gestures on the steering wheel," in Proceedings of the 4th International Conference on Automotive User
Interfaces and Interactive Vehicular Applications, ser. AutomotiveUI '12. New York, NY, USA: ACM, 2012, pp. 155-162. [Online]. Available: http://doi.acm.org/10.1145/2390256.2390282

[12] G. Shakeri, J. H. Williamson, and S. Brewster, "Novel multimodal feedback techniques for in-car mid-air gesture interaction," in Proceedings of the 9th International Conference on Automotive User Interfaces and Interactive Vehicular Applications, ser. AutomotiveUI '17. New York, NY, USA: ACM, 2017, pp. 84-93. [Online]. Available: http://doi.acm.org/10.1145/3122986.3123011

[13] I. Tashev and Y. C. Ju, "Commute ux: Voice enabled in-car infotainment system." Association for Computing Machinery, Inc., September 2009. [Online]. Available: https://www.microsoft.com/en-us/research/publication/commuteux-voice-enabled-in-car-infotainment-system/

[14] A. Bjelkemyr, T. Dukic, R. Owens, T. Falkmer, and H. Lee, "Support systems designed for older drivers to achieve safe and comfortable driving," Journal of Transportation Technologies, vol. 3, pp. 233-240, 2013.

[15] A. Ziakopoulos, A. Theofilatos, E. Papadimitriou, and G. Yannis, "A meta-analysis of the impacts of operating in-vehicle information systems on road safety," IATSS Research, 2019. [Online]. Available: http://www.sciencedirect.com/science/article/pii/S038611121830030X

[16] K. Lipovac, M. Đerić, M. Tešić, Z. Andrić, and B. Marić, "Mobile phone use while driving-literary review," Transportation Research Part F: Traffic Psychology and Behaviour, vol. 47, pp. 132 - 142, 2017. [Online]. Available: http://www.sciencedirect.com/science/article/pii/S1369847817302838

[17] P. Atchley, S. Atwood, and A. Boulton, "The choice to text and drive in younger drivers: Behavior may shape attitude," Accident Analysis \& Prevention, vol. 43, no. 1, pp. $134-142,2011$. [Online]. Available: http://www.sciencedirect.com/science/article/pii/S0001457510002095

[18] I. A. Al-Darrab, Z. A. Khan, and S. I. Ishrat, "An experimental study on the effect of mobile phone conversation on drivers' reaction time in braking response," Journal of Safety Research, vol. 40, no. 3, pp. 185 - 189, 2009. [Online]. Available: http://www.sciencedirect.com/science/article/pii/S0022437509000334

[19] C. S. Dula, B. A. Martin, R. T. Fox, and R. L. Leonard, "Differing types of cellular phone conversations and dangerous driving," Accident Analysis \& Prevention, vol. 43, no. 1, pp. 187 - 193, 2011. [Online]. Available: http://www.sciencedirect.com/science/article/pii/S0001457510002241 